\title{
Water delivery to dry protoplanets by hit-and-run collisions
}

\author{
Christoph Burger ${ }^{1,2}$, Thomas I. Maindl ${ }^{1}$ and Christoph Schäfer ${ }^{2}$ \\ ${ }^{1}$ Department of Astrophysics, University of Vienna, \\ Türkenschanzstraße 17, 1180 Vienna, Austria \\ email: c.burger@univie.ac.at \\ ${ }^{2}$ Institut für Astronomie und Astrophysik, Eberhard Karls Universität Tübingen, \\ Auf der Morgenstelle 10, 72076 Tübingen, Germany
}

\begin{abstract}
Final water inventories of newly formed terrestrial planets are shaped by their collision history. A setting where volatiles are transported from beyond the snowline to habitable-zone planets suggests collisions of very dry with water-rich bodies. By means of smooth particle hydrodynamics (SPH) simulations we study water delivery in scenarios where a dry target is hit by a water-rich projectile, focusing on hit-and-run encounters with two large surviving bodies, which probably comprise about half of all similar-sized collisions (Genda et al. 2017).
\end{abstract}

Keywords. Hydrodynamics, methods: numerical, planets and satellites: formation

\section{Methods}

We base our analysis in this work on existing collision simulations from Burger et al. (2018) and re-examine these data under the aspect of a dry target being hit by a waterrich projectile. The scenarios comprise differentiated, self-gravitating, embryo-sized bodies, consisting of an iron core, a silicate mantle, and a water(ice) shell. For details about the SPH code and the material model we refer the reader to Schäfer et al. (2016) and Burger et al. (2018). Projectile and target have equal initial compositions, $25 \%$ iron, $65 \%$ silicates and a $10 \%$ water mass fraction (wmf). This allows us to track individual contributions from the projectile/target, by tracking the respective SPH particles' origin. For the results presented here we assume a dry target body by tracing only the projectile's water. The differences to a simulation with a really dry target are small (Burger et al. 2018), and should be negligible for the global quantities we are interested in.

\section{Results and discussion}

Post-collision wmf of the initially dry target are plotted in Fig. 1 for various parameter combinations (impact velocity $v / v_{\text {esc }}$, impact angle $\alpha, \gamma=M_{\text {proj }} / M_{\mathrm{targ}}$ ). Typical hit-and-run collisions (top panels) are compared to head-on collisions (bottom panels). Along with that the water accretion efficiency is illustrated, here defined simply as $\xi_{\mathrm{w}}=M_{\mathrm{w}, \mathrm{tf}} / M_{\mathrm{w}, \mathrm{p}}$ with water mass on the target fragment and the projectile (see also Burger \& Schäfer 2017). We abandon the usual notion of the projectile being the smaller body and switch to a one-body perspective, with an always dry target being hit by a (possibly even more massive) waterrich projectile. In our low-velocity hit-and-run scenarios $\left(v / v_{\text {esc }}=1.5, \alpha=45^{\circ}\right.$ in Fig. 1) the target's post-collision wmf is strongly increasing with $\gamma$, and this trend continues even for projectiles considerably larger than the target. This behavior is qualitatively different for higher impact velocities $\left(v / v_{\mathrm{esc}}=\{2.5,3.5\}, \alpha=45^{\circ}\right)$, where the wmf accreted by the target increases with $\gamma$ and peaks at mass ratios of roughly 1:1, before decreasing again, despite the potentially huge water supply in larger projectiles. The water accretion efficiency $\xi_{\mathrm{w}}-$ 


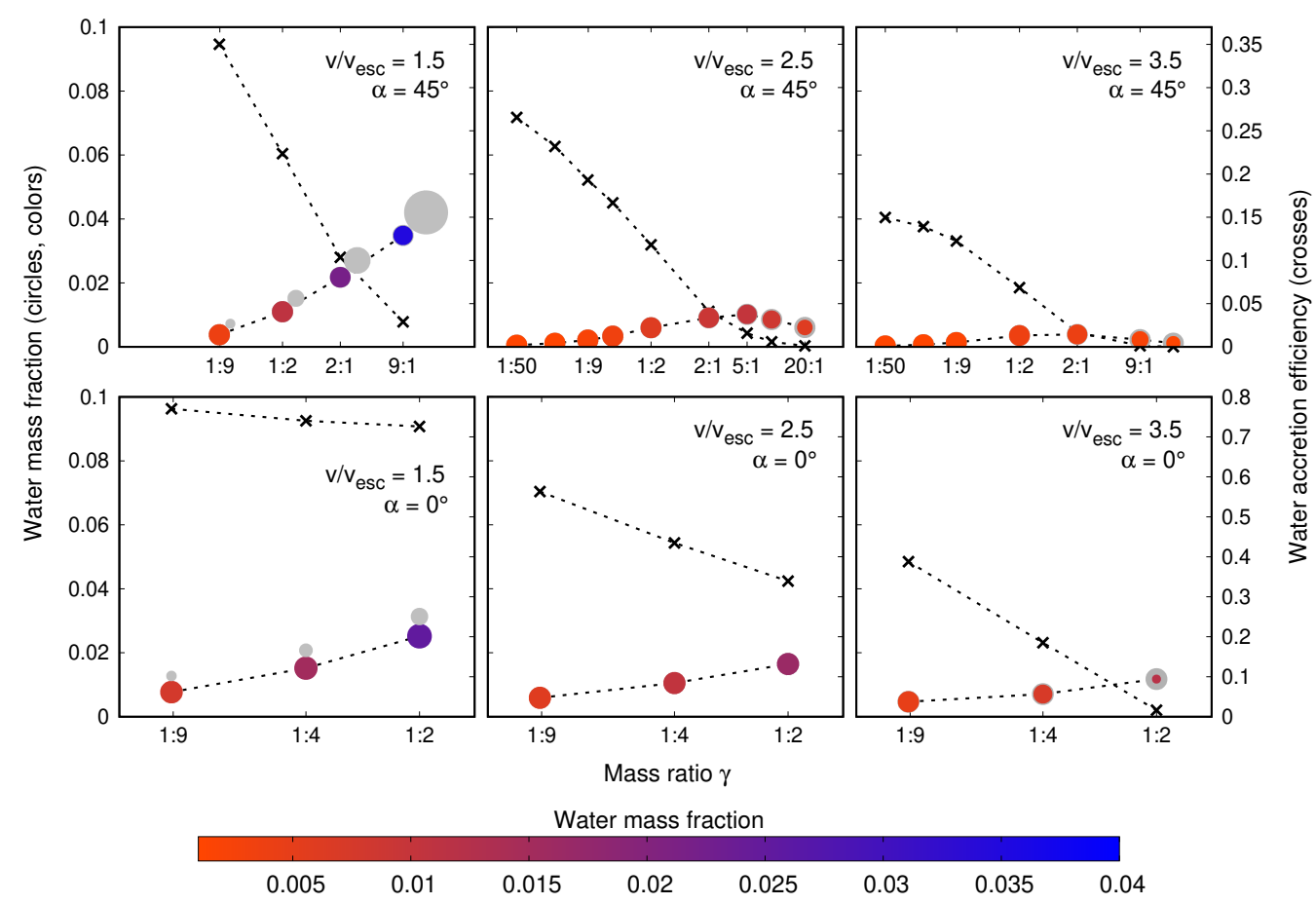

Figure 1. Dry targets being hit by water-rich projectiles $(w m f=0.1)$, for hit-and-run (top row) and head-on collisions (bottom row). Post-collision wmf are plotted on the left y-axes and colorcoded. Water accretion efficiencies $\xi_{\mathrm{w}}$ are plotted on the right y-axes. Grey circles indicate either pre-collision sizes $\left(\propto \operatorname{mass}^{1 / 3}\right)$, or the projectile body (impacting from the top).

the fraction of projectile water accreted by the target - is a decreasing function of $\gamma$ for all scenarios in Fig. 1, and is generally high for small projectile bodies (small $\gamma$ ), but tends to be very low for larger impact velocities and/or mass ratios, indicating that only very little water is transferred to the target.

From the point of view of individual hit-and-run encounters the most water can be delivered to dry target bodies with either low impact velocities, or mass ratios approaching $1: 1$ for higher $v / v_{\text {esc }}$. However, planet formation is a chaotic process and planets grow from a stochastic history of successive collisions. Therefore it will be rather the (average) water accretion efficiency that determines how much water a growing planet can accrete from the limited amount of water-rich material scattered into its feeding zone. Our results show that this figure is considerably higher for smaller hit-and-run projectiles compared to larger ones, and yet significantly higher for head-on collisions, with water retention up to $80 \%$, compared to at most $35 \%$ in our hit-and-run scenarios. It is important to emphasize however, that this does not include the further fate of the (potentially still very water-rich) projectile, after a hit-and-run encounter, therefore it is crucial to consider and track both hit-and-run fragments and their volatile inventories.

\section{References}

Burger, C. \& Schäfer, C. 2017, Proceedings of the First Greek-Austrian Workshop on Extrasolar Planetary Systems, 63

Burger, C., Maindl, T.I., \& Schäfer, C. 2018, CeMDA, 130, 2

Genda, H., Iizuka, T., Sasaki, T., Ueno, Y., \& Ikoma, M. 2017, E\&PSL, 470, 87

Schäfer, C., Riecker, S., Maindl, T.I., Speith, R., Scherrer, S., \& Kley, W. 2016, A $\mho A$ A, 590, A19 